\title{
3-D geometric imaging of the trunk in normal adolescents and age-matched patients impaired with idiopathic scoliosis: selected effects of conservative intervention according to Schroth
}

\author{
S Stoler ${ }^{*}, \mathrm{H}$ Baransi, U Givon, Z Dvir \\ From 9th International Conference on Conservative Management of Spinal Deformities - SOSORT 2012 \\ Annual Meeting \\ Milan, Italy. 10-12 May 2012
}

\section{Background}

Research has indicated that combining the Schroth approach with corrective bracing may lead to a reduction in the number of interventions, and a deceleration in the progression of the scoliotic curve. Yet, it is still unclear whether the postural correction improves the critical factor of trunk symmetry.

\section{Objectives}

To investigate the effectiveness of the Schroth method on trunk symmetry, in standing, sitting, and walking, using 3-D motion pick up (3-DMP) based analysis.

\section{Method}

Twenty three adolescents, (12 healthy and 11 with AIS) participated in the study. All AIS patients had at least one curve of $20^{\circ}$, and were skilled in postural corrections based on Schroth principles. Trunk symmetry was assessed using the Coda Cx1 3DMP system. Markers were applied on the head, acromioclavicular joints, suprasternal notch, scapular inferior angles, pelvis, knee, hip, lateral malleoli and the heads of the 5th metatarsus. All patients were measured twice in the same day, (with a 4-hour break between sessions), in natural sitting, standing, and walking. AIS patients were measured again in those three positions, during postural correction according to Schroth. From the test-retest paradigm the stringent cut-off for a true clinical change - the smallest real difference (SRD) - was derived.

The Institute of Motor Functions, Sheba Medical Center, Ramat-Gan, Israel

\section{Results}

There was a significant and high correlation between the Cobb angle and scapular transverse misalignment. AIS patients showed significant correction of scapular rotation, in standing, and the location of the center of scapulae relative to the center of the pelvis. At least $36 \%$ of the patients corrected the previous, and more than $45 \%$ that of the latter, beyond the SRD. With respect to shifting of the shoulder girdle relative to the pelvis in standing and walking, $55 \%$ of the patients have exceeded the SRD value.

\section{Conclusions}

3DMP analysis enables identification of subtle spinal relationships, which are unrecognizable using visual inspection. Using an advanced 3DMP system, this study confirms the good reproducibility of trunk marking. Moreover, it supports the effectiveness of the Schroth approach in AIS patients who are able to reduce specific trunk asymmetries, particularly those related to shoulder girdle and scapular orientation.

Published: 3 June 2013

References

1. Lexell JE, Downham DY: How to assess the reliability of measurements in rehabilitation. Am J Phys Med Rehabil 2005, 84(9):719-723.

2. Negrini S, Antonini G, Carabalona R, Minozzi S: Physical exercises as a treatment for adolescent idiopathic scoliosis. A systematic review. Pediatr Rehabil 2003, 6(3-4):227-235.

3. Rigo M, Q-S G, Villagrasa $\mathrm{M}$, et al: Various methods of physiotherapy. IOS Press;TB Gravis 2008.

\section{doi:10.1186/1748-7161-8-S1-O20}

Cite this article as: Stoler et al.: 3-D geometric imaging of the trunk in normal adolescents and age-matched patients impaired with idiopathic scoliosis: selected effects of conservative intervention according to Schroth. Scoliosis 2013 8(Suppl 1):O20. 